\title{
Design optimization of ultra-fast silicon detectors
}

\author{
N. Cartiglia ${ }^{\mathrm{a}, *}$, R. Arcidiacono ${ }^{\mathrm{c}}, \mathrm{M}$. Baselga ${ }^{\mathrm{e}}, \mathrm{R}$. Bellan $^{\mathrm{b}}, \mathrm{M}$. Boscardin ${ }^{\mathrm{f}}, \mathrm{F} \cdot$ Cenna $^{\mathrm{a}}$, \\ G.F. Dalla Betta ${ }^{g}$, P. Fernndez-Martnez ${ }^{\text {e }}$, M. Ferrero ${ }^{\mathrm{a}, \mathrm{b}}$, D. Flores ${ }^{\mathrm{e}}$, Z. Galloway ${ }^{\mathrm{d}}$, \\ V. Greco ${ }^{\mathrm{e}}$, S. Hidalgo ${ }^{\mathrm{e}}$, F. Marchetto ${ }^{\mathrm{a}}$, V. Monaco ${ }^{\mathrm{b}}$, M. Obertino ${ }^{\mathrm{c}}$, L. Pancheri ${ }^{\mathrm{g}}$,

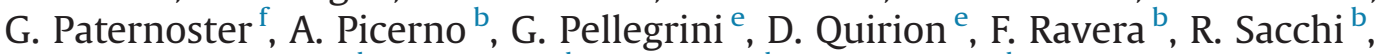 \\ H.F.-W. Sadrozinski ${ }^{\mathrm{d}}$, A. Seiden ${ }^{\mathrm{d}}$, A. Solano $^{\mathrm{b}}$, N. Spencer ${ }^{\mathrm{d}}$ \\ a INFN Torino, Italy \\ ${ }^{\mathrm{b}}$ Università di Torino, Torino, Italy \\ c Università del Piemonte Orientale, Novara, Italy \\ d Santa Cruz Institute for Particle Physics UC Santa Cruz, CA 95064, USA \\ e Centro Nacional de Microeletronica, IMB-CNM, Barcelona, Spain \\ ${ }^{\mathrm{f}}$ Fondazione Bruno Kessler, Via Sommarive 18, 38123 Trento, Italy \\ ${ }^{g}$ Università di Trento, Via Sommarive 9, 38123 Trento, Italy
}

\section{A R T I C L E I N F O}

Keywords:

Silicon

Fast detector

Low gain

APD

Charge multiplication

\begin{abstract}
A B S T R A C T
Low-Gain Avalanche Diodes (LGAD) are silicon detectors with output signals that are about a factor of 10 larger than those of traditional sensors. In this paper we analyze how the design of LGAD can be optimized to exploit their increased output signal to reach optimum timing performances. Our simulations show that these sensors, the so-called Ultra-Fast Silicon Detectors (UFSD), will be able to reach a time resolution factor of 10 better than that of traditional silicon sensors.
\end{abstract}

(c) 2015 Elsevier B.V. All rights reserved.

\section{Introduction}

The design of ultra-fast silicon detectors [1,2] exploits the effect of charge multiplication in LGAD to obtain silicon detectors that can concurrently measure with high accuracy time and space. Low-Gain Avalanche Diodes, as developed by CNM [3], are $n-i n-p$ silicon sensors with a high ohmic $p$ bulk which have a $p^{+}$implant extending several microns underneath the $n$-implant. Fig. 1 shows on the left a schematic of a traditional silicon diode, while on the right the $n^{++}-p^{+}-p-p^{++}$structure of an LGAD. The extra deep $p^{+}$layer creates a strong electric field that generates charge multiplication.

Time resolution: The time resolution $\sigma_{t}$ can be expressed as the sum of three terms [4]: (i) Time Walk, (ii) Jitter, and (iii) TDC binning:

$\sigma_{t}^{2}=\left(\left[\frac{V_{t h}}{S / t_{r}}\right]_{R M S}\right)^{2}+\left(\frac{N}{S / t_{r}}\right)^{2}+\left(\frac{\mathrm{TDC}_{b i n}}{\sqrt{12}}\right)^{2}$

where $S$ is the signal amplitude, $t_{r}$ the signal rise time, $N$ the noise, and $V_{t h}$ is the comparator threshold used to set the time of arrival of the particle $\left(V_{t h} \sim 10 * N\right)$. Eq. (1) shows the first set of

\footnotetext{
* Corresponding author.

E-mail address: cartiglia@to.infn.it (N. Cartiglia).
}

requirements to obtain excellent timing resolution: (i) low noise, (ii) large signals, and (iii) short rise time. The key to excellent time resolution is therefore a large signal $S$ with small rise time $t_{r}$, i.e. we need to maximize the ratio $S / t_{r}$ (or equivalently the slew rate $d V / d t$ ) while keeping the noise $N$ small. These requirements are complemented by the additional request of having signals that are very uniform: if the signal shape changes by a large amount on an event-to event basis, than the timing accuracy is severely degraded.

\section{Signal shape}

In a silicon sensor, an impinging minimum ionizing particle creates electron-hole pairs ( $\sim 75$ electron-holes pairs per micron) that drift toward the electrodes under the influence of an external electric field generated by the bias voltage. The electrons and holes generated by a passing-through particle drift quite rapidly towards the electrodes, reaching a velocity of $100 \mu \mathrm{m} / \mathrm{ns}$ when a sufficiently high field is applied: for typical sensor thicknesses $(200-300 \mu \mathrm{m})$ the entire signal can be collected in 3 ns. This collection time, however, cannot be decreased due to the saturation of the drift velocity $\left(v_{\text {sat }} \sim 10^{7} \mathrm{~cm} / \mathrm{s}\right)$. The shape of the induced current signal can be calculated using Ramo's [5] theorem that states that the current induced by a charge carrier is proportional to its electric charge $q$, the 
drift velocity $v$ and the weighting field $E_{w}$ :

$i \propto q v E_{w}$.

Drift velocity: The drift velocity in silicon sensors is a function of the applied voltage, with a linear dependence at low values of the electric field while it saturates when the field is above $10-20 \mathrm{kV} / \mathrm{cm}$. The need to have signals with fast rise time and uniform shapes requires to operate UFSD where the velocity is saturated, and therefore the sensor design should be such that a large external potential can be applied without causing electric breakdown. This requirement also implies that UFSD needs to use very high resistivity silicon so that the electric field is as uniform as possible.

Weighting field: The weighting field $E_{w}$ describes the coupling of the charge carriers to the read-out electrode and it depends uniquely on the geometry of the sensor. The best possible weighting field is obtained for geometries similar to that of a parallel plate capacitor, while highly segmented sensors suffer from a strongly varying $E_{w}$. The values of $E_{w}$ for two different strip geometries are shown in Fig. 2: a $300 \mu \mathrm{m}$ pitch and a $50 \mu \mathrm{m}$ implant on the left side and a $300 \mu \mathrm{m}$ pitch and a $290 \mu \mathrm{m}$ implant on the right side. The white dashed lines are the pitch boundaries. Since the particles are crossing the sensor perpendicularly, the weighting field should be the same for any track crossing the $x$ axis perpendicularly, which is clearly not the case in the left panel of Fig. 2.

Signal amplitude in silicon sensors without gain: Using Ramo's theorem we can calculate the maximum current in a pad detector of thickness $d$, assuming a saturated drift velocity $v_{\text {sat }}$ :

$I_{\text {max }} \propto N q \frac{1}{d} v_{\text {sat }}=75 d q \frac{1}{d} v_{\text {sat }}=75 q v_{\text {sat }}$

where $E_{w} \propto 1 / d$ is the weighting field for a pad geometry and $N$ is the number of e/h pairs $(N=75 \mathrm{~d})$. This result shows an interesting feature of silicon sensors: the peak current does not depend on the sensor thickness. Thick sensors have indeed a larger number $(N)$ of
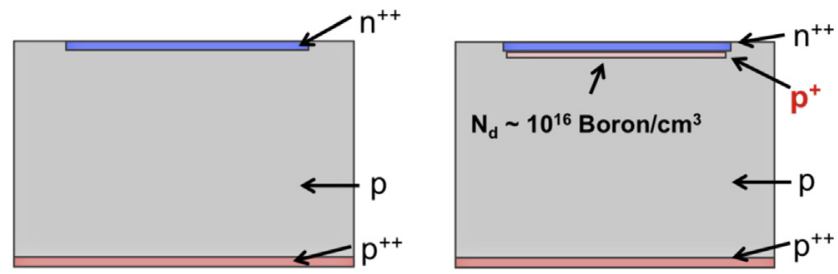

\section{Traditional Silicon Diode Low Gain Avalanche Diode}

Fig. 1. Schematic of a traditional silicon diode (left) and of a Low-Gain Avalanche Diode (right). initial e/h pairs, however each pair generates a lower initial current (the weighting field is inversely proportional to the sensor thickness d), Fig. 3. This cancellation is such that the peak current in silicon detectors is always the same, $I_{\max } \sim 1-2 \mu \mathrm{A}$, regardless of the sensor thickness and therefore the time resolutions of thin and thick sensors are very similar.

\section{Charge multiplication in silicon sensors}

Charge multiplication in silicon sensors happens when the charge carriers are in electric fields of the order of $E \sim 300 \mathrm{kV} / \mathrm{cm}$. Under this condition the electrons (and to less extent the holes) acquire sufficient kinetic energy that are able to generate additional e/h pairs. A field value of $300 \mathrm{kV} / \mathrm{cm}$ is not reachable applying an external voltage $V_{\text {Bias }}$ without causing electrical breakdown, but it is obtained by implanting an appropriate charge density that locally generates very high fields $\left(N_{D} \sim 10^{16} / \mathrm{cm}^{3}\right)$. The gain has an exponential dependence on the electric field $N(l)=N_{o} e^{\alpha(E) l}$, where $\alpha(E)$ is a strong function of the electric field and $l$ is the path length inside the high field region. The additional doping layer present at the $n-p$ junction in the LGAD design, Fig. 1, generates the high field necessary to achieve charge multiplication.

\section{The Weightfield2 simulation program}

We have developed a full simulation program, Weightfield 2 (WF2), this issue, [6] with the specific aim of assessing the timing capability of silicon and diamond sensors.

This program uses GEANT4 [7] libraries to simulate the energy released by an impinging particle in silicon (or diamond), and Ramo's theorem to generate the induced signal current. The program has a graphical user interface, shown in Fig. 4, that
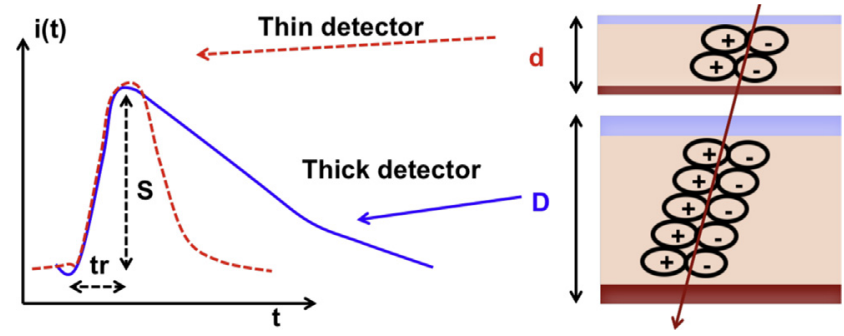

Fig. 3. The initial signal amplitude in silicon sensors does not depend on their thickness: thin and thick detectors have the same maximum current, and thick detectors have longer signals.
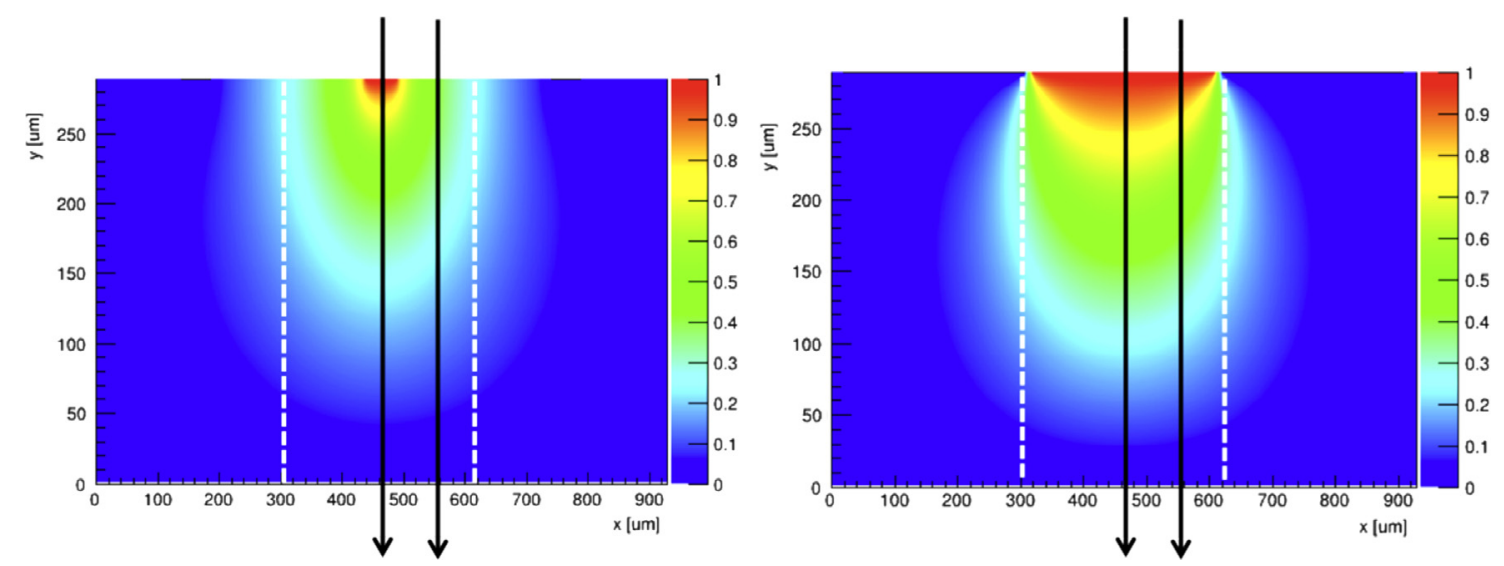

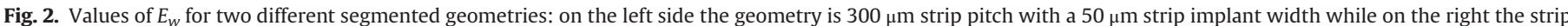
implant is $290 \mu \mathrm{m}$. 


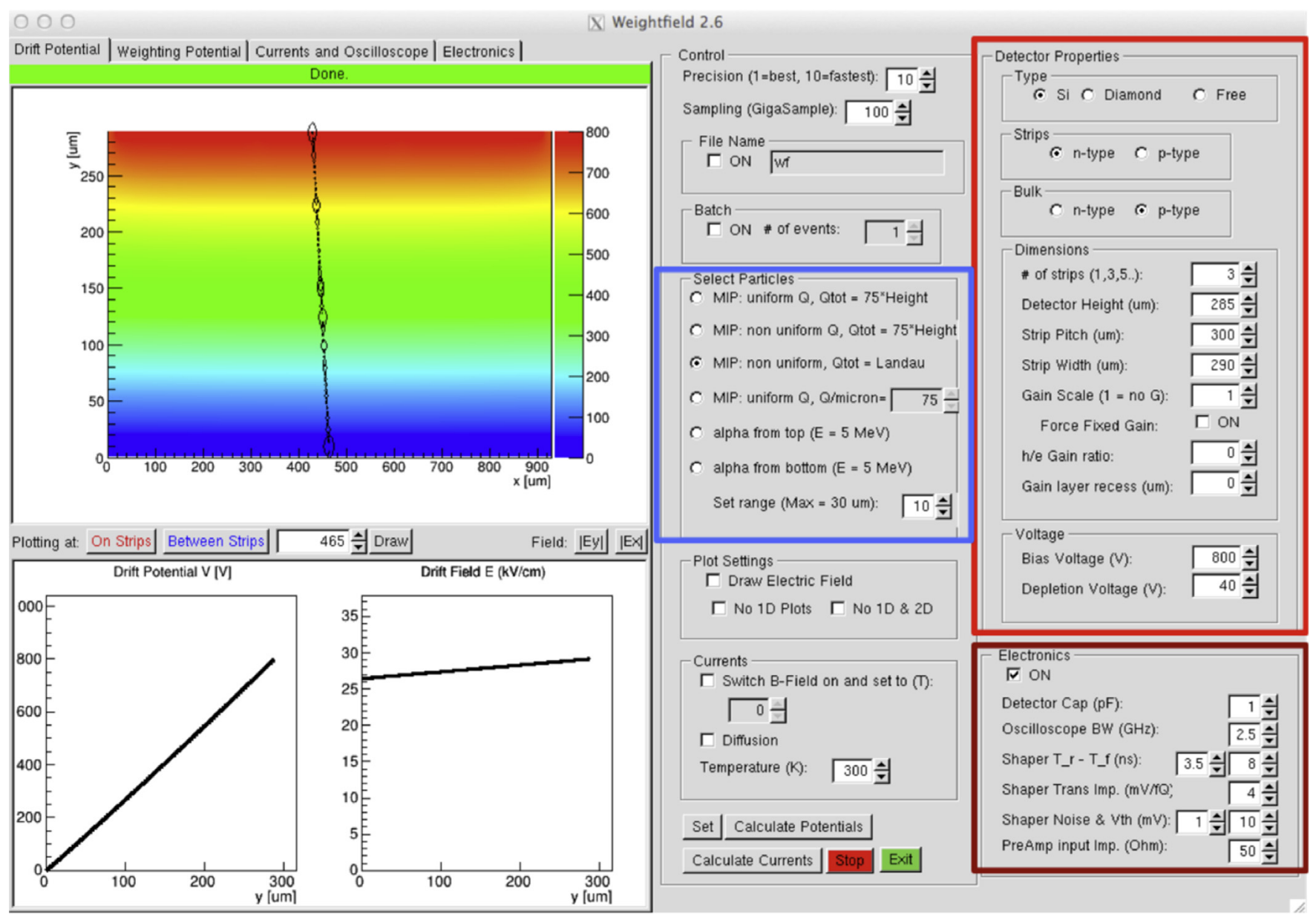

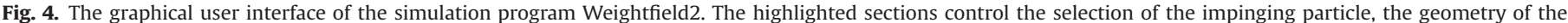
sensor and the parameters of the read-out electronics.

allows configuring many input parameters such as (i) incident particle, (ii) sensor geometry, (iii) presence and value of internal gain, (iv) doping of silicon sensor and its operating conditions, (v) the values of an external B-field, ambient temperature and thermal diffusion and finally (vi) the oscilloscope and front-end electronics response. The program has been validated comparing its predictions for minimum ionizing and alpha particles with measured signals and TCAD simulations, finding excellent agreement in both cases. All the subsequent simulation plots and field maps shown in this paper have been obtained with WF2.

\section{Optimization of UFSD sensors}

\subsection{The effect of charge multiplication}

Using WF2 we can simulate the output signal of UFSD sensors as a function of many parameters, such as the gain value, sensor thickness, electrode segmentation, and external electric field. Fig. 5 shows the simulated current, and its components, for a $50-\mu \mathrm{m}$ thick detector. The initial electrons (red), drifting toward the $n++$ electrode, go through the gain layer and generate additional $\mathrm{e} / \mathrm{h}$ pairs. The gain electrons (violet) are readily absorbed by the cathode while the gain holes (light blue) drift toward the anode and they generate a large current.

The gain dramatically increases the signal amplitude, producing a much higher slew rate. The value of the current generated by a gain $G$ can be estimated in the following way: (i) in a given time interval $d t$, the number of electrons entering the gain region

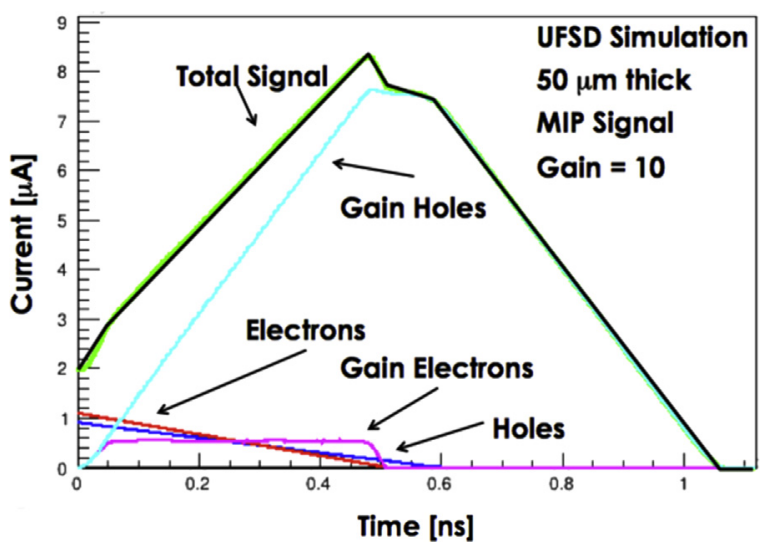

Fig. 5. UFSD simulated current signal for a $50-\mu \mathrm{m}$ thick detector. (For interpretation of the references to color in this figure caption, the reader is referred to the web version of this paper.)

is $75 v d t$ (assuming $75 \mathrm{e} / \mathrm{h}$ pairs per micron) and (ii) these electrons generate $d N_{\text {Gain }} \propto 75 v d t G$ new e/h pairs. Using again Ramo's theorem, the current induced by these new charges is given by

$d i_{\text {Gain }}=d N_{\text {Gain }} q v_{\text {sat }} \frac{k}{d} \propto \frac{G}{d} d t$ 
which leads to the following expression for the slew rate:

$\frac{d i_{\text {Gain }}}{d t} \sim \frac{d V}{d t} \propto \frac{G}{d}$.

Eq. (5) demonstrates a very important feature of UFSD: the slew rate increase due to the gain mechanism is proportional to the ratio of the gain value over the sensor thickness $(G / d)$, therefore thin detectors with high gain provide the best time resolution. Specifically, the maximum signal amplitude is controlled only by the gain value, while the signal rise time only by the sensor thickness, Fig. 6.

Using WF2 we have cross-checked this prediction simulating the slew rate for different sensors thicknesses and gains, Fig. 7: the slew rate in thick sensors, $200-$ and $300-\mu \mathrm{m}$, is a factor of $\sim 2$ steeper than that of traditional sensors, while in thin detectors, 50 - and $100-\mu \mathrm{m}$ thick, the slew rate is 5-6 times steeper. For gain $=1$ (i.e. traditional silicon sensors) WF2 confirms the predictions of Eq. (3): the slew rate does not change as a function of thickness.

\subsection{Segmented read-out and gain layer position}

As stated above, excellent timing capability requires very uniform fields and gain values however this fact might be in contradiction with the goal of having finely segmented electrodes.

There are 4 possible relative positions of the gain layer with respect to the segmented read-out electrodes, depending on the type of the silicon bulk and strip, Fig. 8. For $n-i n-p$ detectors (top left), the gain layer is underneath the read-out electrodes, while it is on the opposite side of the read-out electrodes in the $p-i n-p$ design (bottom left). Likewise, for $p-i n-n$ sensors the gain layer is at the read-out electrodes, while it is on the opposite side for $n-i n-n$

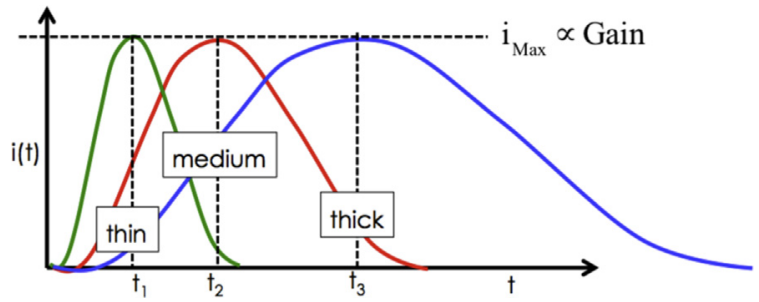

Fig. 6. In UFSD the maximum signal amplitude depends only on the gain value, while the signal rise time only on the sensor thickness: sensors of 3 different thicknesses (thin, medium, thick) with the same gain have signals with the same amplitude but with different rise time.

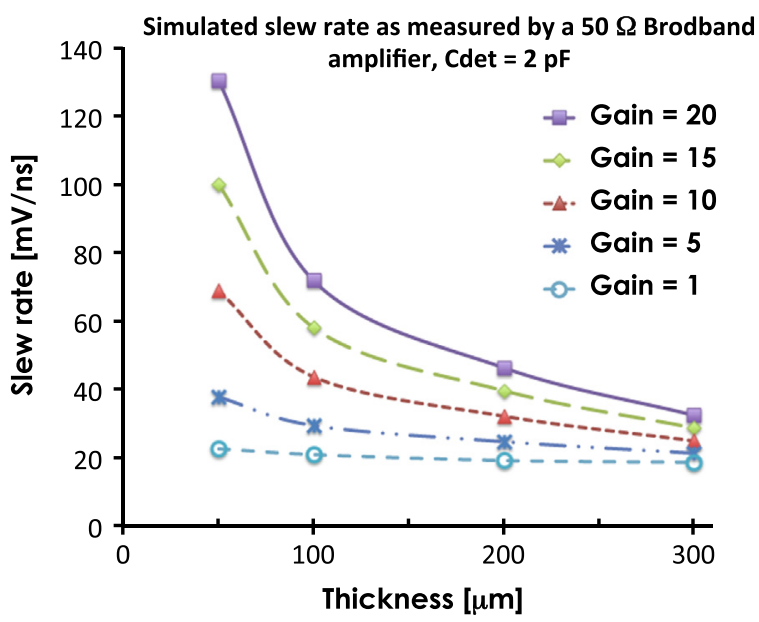

Fig. 7. Simulated UFSD slew rate as a function of gain and sensor thickness. Thin sensors with even moderate gain (10-20) achieve a much higher slew rate than traditional sensors (gain $=1$ ).

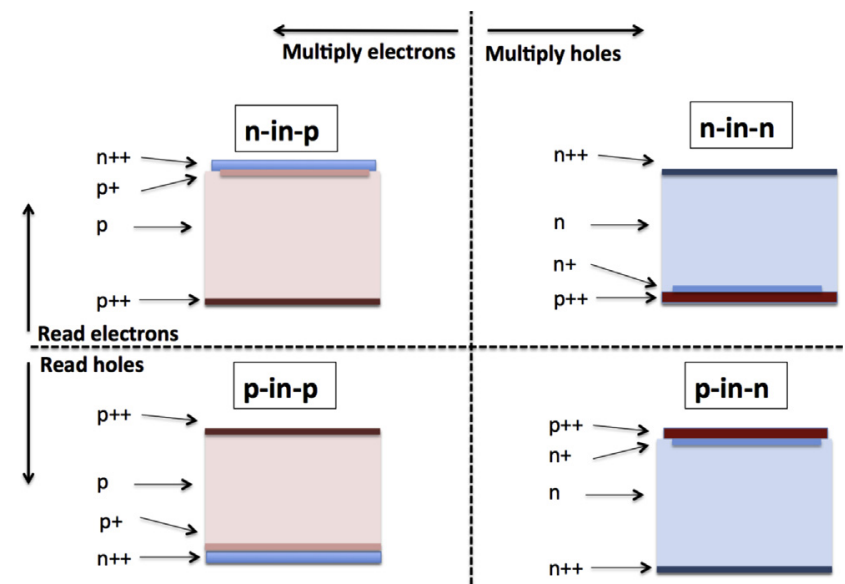

Fig. 8. 4 possible configurations of the gain layer. In n-bulk sensors the multiplication is initiated by holes, while in p-bulk sensors by electrons.

sensors (bottom right). The use of n-bulk sensors presents however a very challenging problem: for this geometry, the multiplication mechanism is initiated by the drifting holes, and therefore is much harder to control as it tends to rapidly evolve into Geiger mode. We have therefore decided not to purse this possibility any further. Fig. 9 shows the potential fields for the $n-i n-p$ and $p-i n-p$ geometries when the read-out is highly segmented.

Before deciding between the $n-i n-p$ and the $p-i n-p$ designs we need to consider also the effect of the weighting field on the signal shape: in segmented detectors the weighting field is such that only charges $(\mathrm{e} / \mathrm{h})$ near the read-out electrode contribute significantly to the signal. Fig. 10 shows this effect: on the left side there are the current signals from a minimum ionizing particle in an $n-i n-p$ (top) and in a $p-i n-p$ (bottom) $300 \mu \mathrm{m}$ thick sensor while on the right the equivalent signals from $100 \mu \mathrm{m}$ thick sensors. In thick detectors, the signal from a $p-i n-p$ sensor (bottom left) is severely delayed with respect to the $n-i n-p$ signal (top left) and it has a shape that cannot be used effectively for timing determination. Conversely, in thin detectors (right side) the current signals are rather similar as one would expect for an almost uniform weighting field.

We can therefore conclude that UFSD should be based on $n-i n-p$ sensors for applications that allows for large size electrodes, while it should be based on thin $p-i n-p$ sensors for applications requiring highly segmented read-out electrodes.

\subsection{The effect of Landau fluctuations}

The final limit to signal uniformity is given by the physics governing energy deposition in silicon: the charge distribution created by an ionizing particle crossing the sensor varies on an event-by-event basis. These variations not only produce an overall change in signal magnitude, which is at the root of the time walk effect, but also produce a more irregular current signal. The left picture in Fig. 11 shows the simulated energy deposition of a minimum ionizing particle, while the right picture the generated current signal and its components. As the picture shows, the variations are rather large and they can severely degrade the achievable time resolution. There are two ways to mitigate this effect: (i) integrating the output current over times longer than the typical spike length and (ii) using thin sensors, as their steeper signal is more immune to signal fluctuations.

\section{Optimization of UFSD read-out electronics}

The ultimate performance of UFSD depends critically on the combination of sensors and read-out electronics. A highly pixelated 


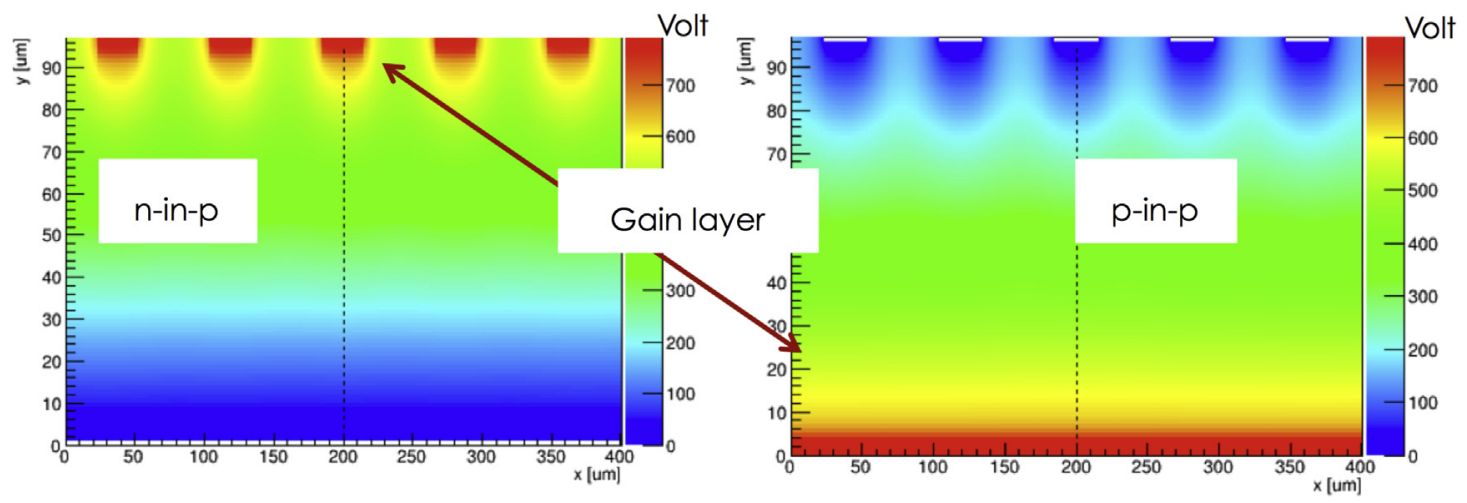

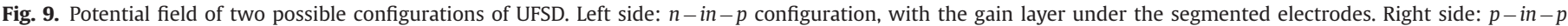
configuration with the gain layer in the deep side. The secondary $y$-axis shows the value of the potential.
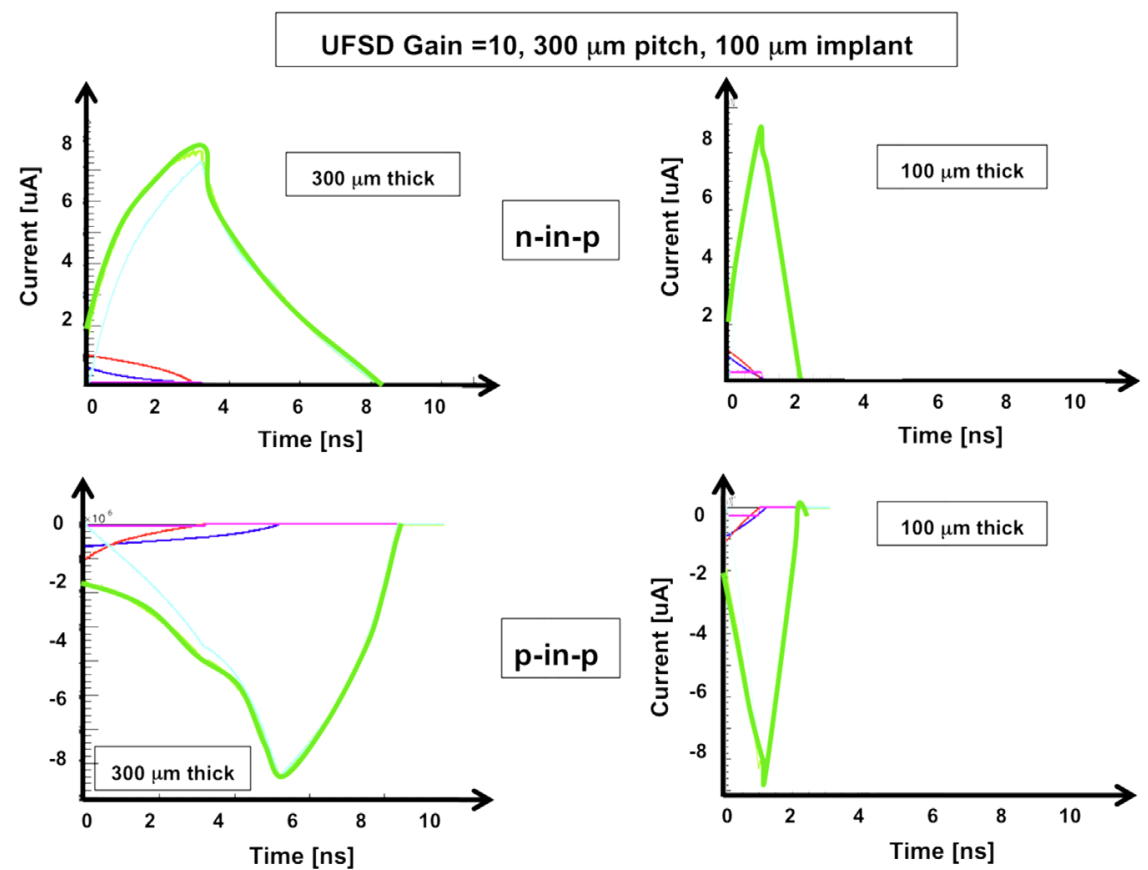

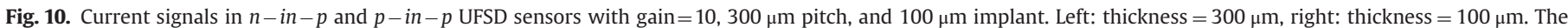

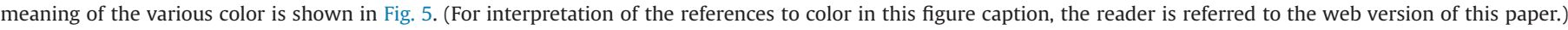

UFSD requires a full custom ASIC read-out, bump bonded to the sensor. Even though the details of the read-out design will depend on the specific technological choices, we outline here several general issues.

\subsection{Interplay of signal rise time, detector capacitance and read-out input impedance}

The charges collected on the read-out electrode of the sensor move to the input of the read-out electronics with a time constant $\tau$ given by the product of the detector capacitance $C_{d e t}$ and the read-out input impedance $R_{i n}: \tau=R_{i n} C_{\text {det }}$, Fig. 12 .

In order to fully exploit the very high slew rate offered by UFSD, $\tau$ has to be shorter or, at most, of the same order of the signal rise time, $t_{\text {rise. }}$. This constrain is strongly linking sensor and electronics designs, as the electronics should be such that it does not slow down very fast input signals. For example, pre-amplifiers that use SiGe technologies tend to have higher input impedance (100$300 \mathrm{Ohm}$ ) and therefore can be coupled only to small sensors $\left(C_{\text {Det }}<2 \mathrm{pF}\right)$, so that the value of $\tau$ remains below $t_{\text {rise }}$ $\left(t_{\text {rise }} \sim 500 \mathrm{ps}\right.$ for a $50 \mu \mathrm{m}$ thick sensor). Our simulations indicate that large values of $\tau$ have indeed negative effects on the slew rate, but they have beneficial effects in smoothing out the Landau fluctuations, and we have identified that the best compromise between these two effects is achieved when $\tau \sim t_{\text {rise }}$.

\subsection{Choice of preamplifier architecture}

We have considered two possible pre-amplifier designs: (i) current amplifiers (CA) or (ii) charge sensitive amplifiers (CSA). With CA the signals are amplified without strong additional shaping while with CSA the signals are integrated and shaped. There are several issues that need to be considered when using either approach: CAs are much faster, and they are able to take full advantage of the very fast signal slew rate but they have a higher noise, while CSA are somewhat slower but the integration they perform makes the output signal more immune to noise and Landau fluctuations. The choice between these two architectures needs to be evaluated in conjunction with the sensor dimensions since if the unavoidable signal integration due to the detector capacitance is enough to smooth out the effect of Landau 

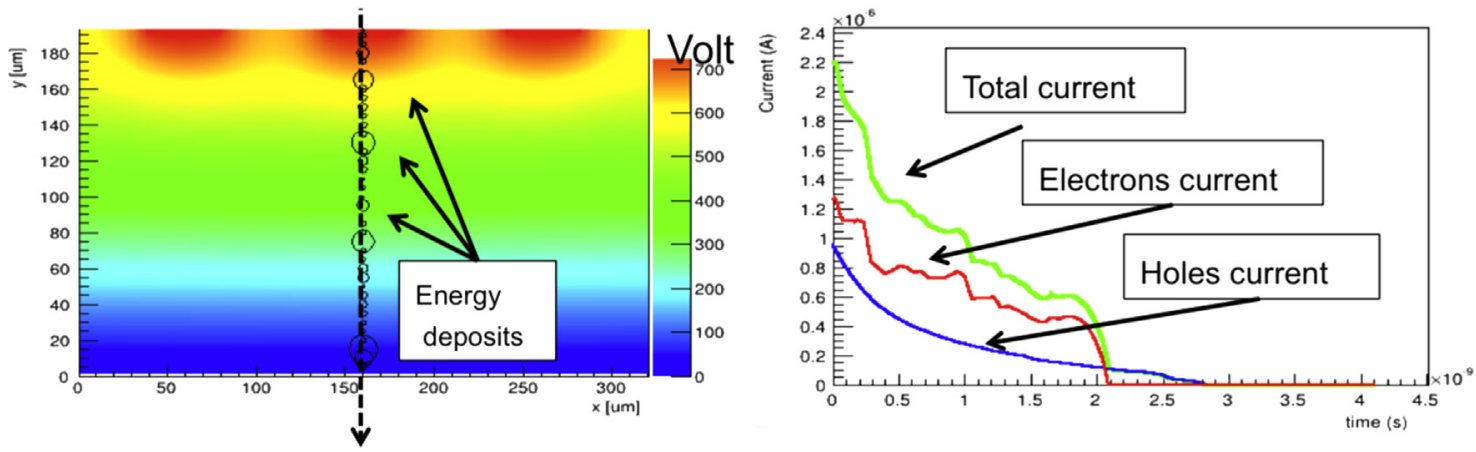

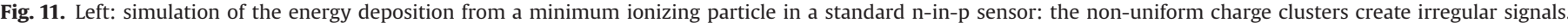
Right: the current signal associated with the clusters shown on the left side.

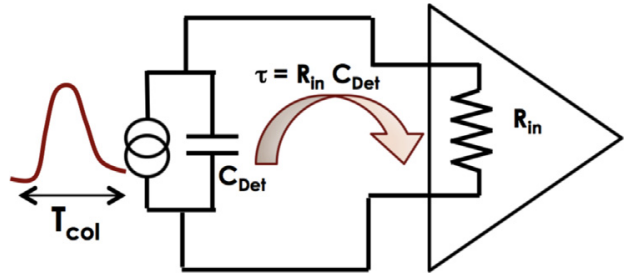

Fig. 12. Interplay of the signal rise time, detector capacitance and read-out input impedance.

fluctuations, then CA will provide the best results while if this is not the case then the second integration offered by the CSA is needed.

\subsection{The effect of gain on the electronic noise}

As Eq. (1) indicates, time resolution is directly proportional to the system noise $N$. Fig. 13 shows on the left side the physical configuration of a sensor with its front-end pre-amplifier, while on the right side the equivalent noise model. The sensor is represented by an ideal capacitor with a current source in parallel, the biasing circuit by a resistor and a current source, while the components leading to the pre-amplifiers are modelled by a series resistor and a voltage source. The full expression of the equivalent noise charge is given by [8]

$$
\begin{aligned}
Q_{n}^{2}= & \left(2 e I_{\text {Det }}+\frac{4 k T}{R_{\text {Bias }}}+i_{N_{A} m p}^{2}\right) F_{i} T_{S} \\
& +\left(4 k T R_{S}+e_{N_{\text {Amp }}}^{2}\right) F_{v} \frac{C_{D e t}^{2}}{T_{S}}+F_{v f} A_{f} C_{D e t}^{2}
\end{aligned}
$$

where the meaning of most of the terms is shown in Fig. 13, $F_{i, v}, A_{f}$ are values close to unity, and $T_{s}$ is the electronics shaping time. The only term that is directly affected by the gain mechanism is the first one of Eq. (6), $Q_{\text {shot }}=2 e I_{\text {Det }}$, that represents the shot noise due to the leakage current going through the $n-p$ junction. As the leakage current follows the same path of the signal, its contribution to the noise increases linearly with the gain value $G: Q_{\text {shot }}=2 e I_{\text {Det }} \rightarrow 2 e G I_{\text {Det }}$. There are several possible mitigation techniques: (i) keep the sensor small, to reduce the absolute value of $I_{\text {Det }}$, (ii) choose the integration time $T_{S}$ short, so that the second term (the so called voltage term) dominates, and (iii) keep the gain value small. A second source of noise directly linked to the gain mechanism is the Excess Noise Factor, which represents the extra noise generated by the multiplication mechanism:

$\mathrm{ENF}=k G+\left(2-\frac{1}{G}\right)(1-k)$ where $G$ is the gain value and $k$ the ratio between the hole and the electron ionization coefficient [9]. The value of ENF depends on the gain $G$, which needs to be kept low, and the term $k$, that can be controlled by carefully designing the doping layer.

\subsection{Choice of time-walk correction circuits}

Time-walk, the unavoidable process by which larger signals cross a given threshold earlier than smaller ones, needs to be corrected by an appropriate electronic circuit. The three most common solutions are illustrated in Fig. 14: (a) Constant Fraction Discriminator (CFD), which sets the time of arrival of a particle when the signal reaches a given fraction of the total amplitude, (b) Time over Threshold (ToT), that uses two time points to evaluate the amplitude of the signal, and apply a correction amplitude-dependent to the first time point $t_{1}$ and (c) Multiple Samplings (MS), where the signal is sampled multiple times, and a fit is used to define the particle time. CFD and ToT are simpler solutions, and they can be implemented per pixel within the readout chip. MS is instead a rather complex algorithm as it requires the full digitization of the signal: this solution gives the best performance, but it can be used only for systems with a limited number of pixels as it needs a fair amount of computing power.

\section{System design}

The design of UFSD requires the optimization of many interrelated parameters. We are considering two distinct options for the realization of a highly pixelated UFSD system, Fig. 15: (i) left: a single read-out chip, able to measure position and time, or (ii) right: a split design, where we use double side read-out to separate the position measurement from the time determination. This second design is mechanically more challenging, however reduces the complexity of each read-out chip. Both designs assure (i) excellent timing capability, due to the enhanced signal and reduced collection time, and (ii) accurate position determination, due to the pixelated electrodes.

\section{Design validation}

The ultimate performance of a UFSD system can only be achieved with the design of VLSI electronics coupled to pixels with small capacitance, as shown in Fig. 15. Large size sensors are however very useful to validate the design choices. Fig. 16 shows the simulated time resolution for a series of 4 sensor prototypes (all with $C_{\text {Det }}=2 \mathrm{pF}$ ) of different thicknesses, read-out by 3 types of electronics front-end that can be designed using discrete 
components. For reference, the empty square and circle show the performance of silicon sensors without internal multiplication. A $300-\mu \mathrm{m}$ thick UFSD with gain 10 will roughly half the time resolution of a standard sensor, and for a UFSD $50-\mu \mathrm{m}$ thick the precision will double again.

\section{Summary}

In this paper we have reviewed the key aspects of the design of UFSD detectors. We list here our main conclusions: (i) UFSD timing performances depend on the ratio of the gain over the sensor thickness and, for gain values of $G \sim 10-15,50 \mu \mathrm{m}$ thick UFSD improve the time resolution of traditional sensors by a factor of $\sim 5$. (ii) The signal amplitude is controlled uniquely by the gain value, while the signal rise time by the sensor thickness. (iii) UFSD can only use p-bulk silicon since the multiplication mechanism needs to be initiated by the electrons. (iv) Highly segmented UFSD can be obtained by positioning the read-out electrodes and the gain layer on opposite side of the sensor, using a $p-i n-p$ design. (v) The effect of Landau fluctuations is controlled by integrating the current signal with a time constant of similar value than the signal rise time. (vi) The product of the sensor capacitance and the read-out electronics input impedance should not be much larger than the signal rise time. (vii) The noise increase due to the added gain depends on the value of the leakage current and the excess noise factor: the first term can be controlled using small sensors while the second term by a careful design of the gain layer.

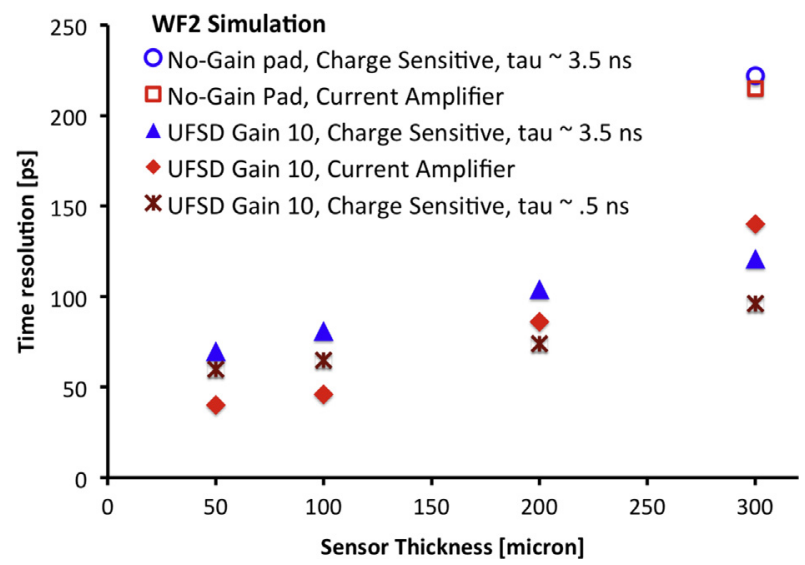

Fig. 16. Simulated time resolutions for a sequence of prototypes read-out using discrete components electronics.

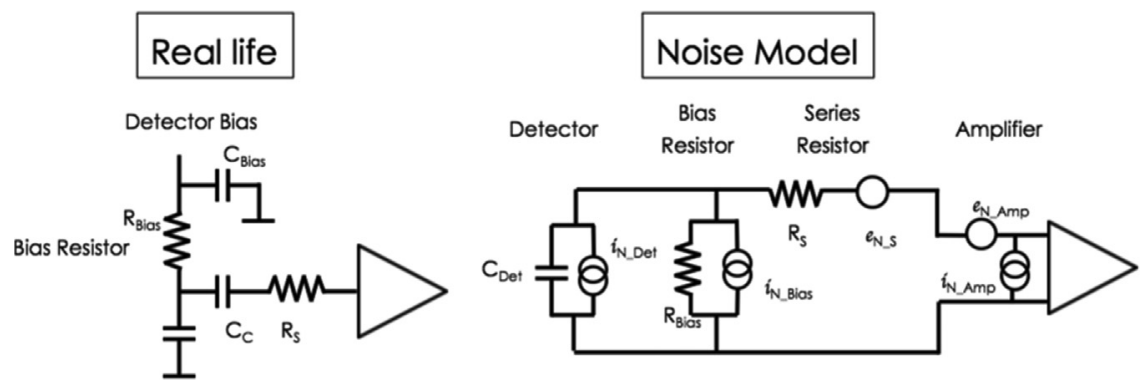

Fig. 13. Right: noise model of the real life sensor-electronics configuration shown on the left.

(a)

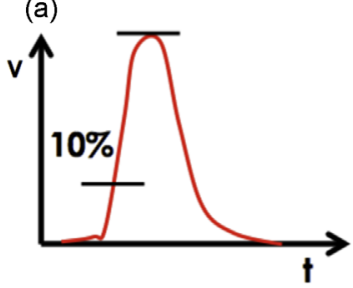

(b)

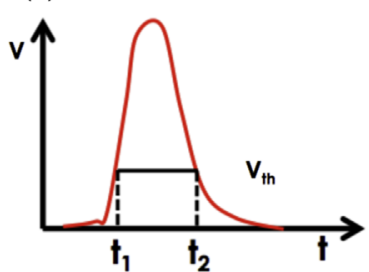

(c)

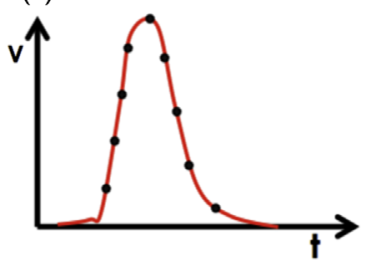

Fig. 14. Time-walk correction techniques: (a) Constant fraction Discriminator, (b) Time Over Threshold, (c) Multiple Samplings.
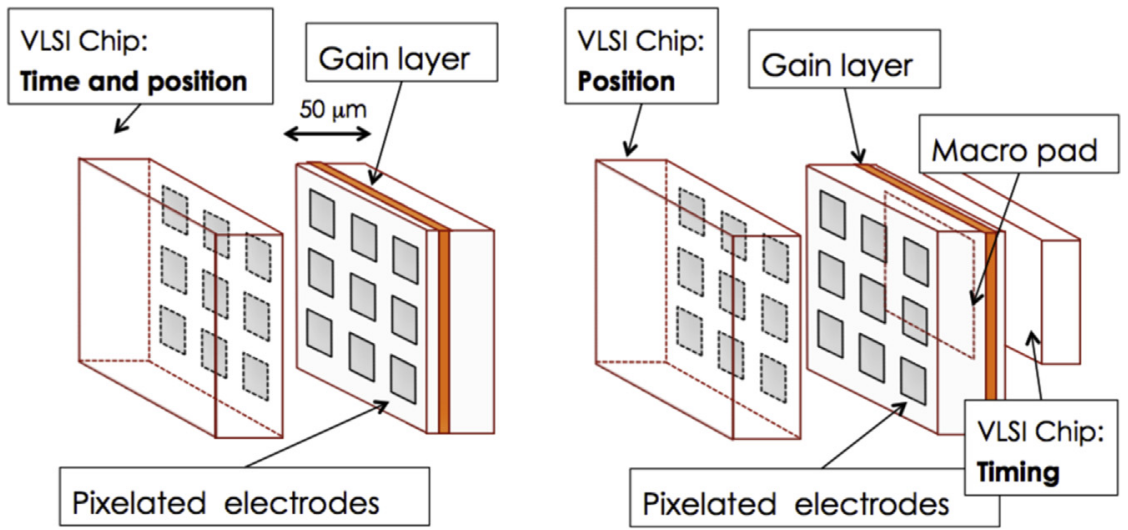

Fig. 15. Sketch of a UFSD sensor and associated VLSI electronics. Left side: single read-out chip, right side: split read-out. 


\section{References}

[1] H.-W. Sadrozinski, et al., Nuclear Instruments and Methods in Physics Research Section A 730 (2013) 226. http://dx.doi.org/10.1016/j.nima.2013.06.033.

[2] H.-W. Sadrozinski, et al., Nuclear Instruments and Methods in Physics Research Section A 765 (2014) 7. http://dx.doi.org/10.1016/j.nima.2014.05.006.

[3] G. Pellegrini, et al., Nuclear Instruments and Methods in Physics Research Section A 765 (2014) 12. http://dx.doi.org/10.1016/j.nima.2014.06.008.

[4] N. Cartiglia, et al., Journal of Instrumentation 9 (2014) C02001. http://dx.doi. org/10.1088/1748-0221/9/02/C02001 arXiv:1312.1080.
[5] S. Ramo, Proceedings of IRE 27 (1939) 584. http://dx.doi.org/10.1109/ JRPROC.1939.228757.

[6] F. Cenna, et al., Nuclear Instruments and Methods in Physics Research Section A RESMDD14, this issue, NIMA57673. http://dx.doi.org/10.1016/j.nima.2015.04. 015.

[7] S. Agostinelli, et al., Nuclear Instruments and Methods in Physics Research Section A 506 (2003) 250. http://dx.doi.org/10.1016/S0168-9002(03)01368-8.

[8] H. Spieler, Semiconductor Detector System, Oxford University Press, Orford (2005) http://dx.doi.org/10.1093/acprof:oso/9780198527848.001.0001.

[9] R. McIntyre, IEEE Transactions on Electron Devices ED-13 (1) (1966) 164. http: //dx.doi.org/10.1109/T-ED.1966.15651. 